\title{
GESTÃO DE RISCOS E ENVELHECIMENTO POPULACIONAL NA IMPLEMENTAÇÃO DA INDÚSTRIA 4.0 NO BRASIL
}

\author{
Stella Cipriano Queiroz \\ Universidade Federal do Rio de Janeiro \\ COPPE/UFRJ - Engenharia de Produção - Sala F-103 - \\ Caixa Postal: 68507 CEP: 21941-972 - Rio de Janeiro RJ \\ stella@ufrj.br \\ Mariana Marins Sant'Anna \\ Universidade Federal do Rio de Janeiro \\ COPPE/UFRJ - Engenharia de Produção - Sala F-103 - \\ Caixa Postal: 68507 CEP: 21941-972 - Rio de Janeiro RJ \\ marianam.santanna@hotmail.com \\ Washington Barbosa \\ Universidade Federal do Rio de Janeiro \\ COPPE/UFRJ - Engenharia de Produção - Sala F-103 - \\ Caixa Postal: 68507 CEP: 21941-972 - Rio de Janeiro RJ \\ washington.fiocruz@gmail.com \\ Leonardo Luciano Souza \\ Universidade Federal do Rio de Janeiro \\ COPPE/UFRJ - Engenharia de Produção - Sala F-103 - \\ Caixa Postal: 68507 CEP: 21941-972 - Rio de Janeiro RJ \\ leonardolucioano@id.uff.br \\ Tharcisio Cotta Fontainha \\ Universidade Federal do Rio de Janeiro \\ COPPE/UFRJ - Engenharia de Produção - Sala F-103 - \\ Caixa Postal: 68507 CEP: 21941-972 - Rio de Janeiro RJ \\ tharcisio@pep.ufrj.br
}

\begin{abstract}
RESUMO
O termo Indústria 4.0 remete a avanços tecnológicos fundamentais a serem implementados no que podemos chamar de $4^{\mathrm{a}}$ Revolução Industrial. Os principais impactos da Indústria 4.0 ocorrem na força de trabalho e na necessidade de as pessoas aperfeiçoarem suas competências para lidar com todas as novas tecnologias. Sabe-se que o Brasil passa por um processo de transição demográfica em que as tendências das taxas brutas de natalidade e mortalidade acarretarão, no início dos anos 2040, a redução no volume populacional. As transformações na dinâmica demográfica impactarão não só o volume, mas também a estrutura etária e, consequentemente, a população em idade ativa, com a redução no número de crianças e o aumento no segmento idoso. A metodologia de Gestão de Riscos é recomendada para que as organizações a incorporem aos seus respectivos planejamentos, subsidiando a tomada de decisão em todos os departamentos e atividades, incluindo estratégias, operações, processos, funções, projetos, produtos, serviços e ativos. O presente
\end{abstract}


artigo tem como objetivo compreender e avaliar os impactos que esta dinâmica pode causar na implementação do modelo de Indústria 4.0 no Brasil, através da utilização de um exemplo de matriz de riscos obtida do PMBOK.

Palavra-chave: Indústria 4.0; Gestão de Riscos; Envelhecimento Populacional; Matriz de Riscos.

\begin{abstract}
The term Industry 4.0 refers to fundamental technological advances to be implemented in what we may call the 4th Industrial Revolution. Industry 4.0's key impacts come from the workforce and the need for people to hone their skills to deal with all new technologies. It is known that Brazil is undergoing a demographic transition process in which trends in gross birth and death rates will lead to a reduction in population volume in the early 2040s. Changes in demographic dynamics will impact not only volume but also age structure and, consequently, the working age population, with the reduction in the number of children and the increase in the elderly segment. The Risk Management methodology is recommended for organizations to incorporate into their planning, supporting decisionmaking across all departments and activities, including strategies, operations, processes, functions, projects, products, services and assets. This article aims to understand and evaluate the impacts this dynamic may cause in the implementation of the Industry 4.0 model in Brazil, using an exemple of risk matrix metodology from PMBOK.
\end{abstract}

Keywords: Industry 4.0; Risk Management; Population Aging; Risk Matrix.

\title{
Como Citar:
}

QUEIROZ, S.Q.; SANT’ANNA, M.M.; BARBOSA, W.; SOUZA, L. L.; FONTAINHA, T.C. Gestão de riscos e envelhecimento populacional na implementação da indústria 4.0 no Brasil. In: SIMPÓSIO DE PESQUISA OPERACIONAL E LOGÍSTICA DA MARINHA, 19., 2019, Rio de Janeiro, RJ. Anais [...]. Rio de Janeiro: Centro de Análises de Sistemas Navais, 2019.

\section{INTRODUÇÃO}

Em 2013, o governo da Alemanha apresenta o relatório de um grupo de trabalho composto pelo Ministério de Educação e Pesquisa e pela Academia Europeia de Administração Industrial, cujo objeto de estudo é a implementação do programa High Tech Strategy 2020 Action Plan. O programa é denominado Industrie 4.0, fazendo referência à $4^{\mathrm{a}}$ Revolução Industrial (Kagermann;Wahlster e Helbig, 2013).

Conforme o relatório, num futuro próximo, as empresas devem estabelecer redes globais que incorporem máquinas, sistemas de armazenagem e unidades de produção na forma de Sistemas Físico-Cibernéticos. Esses são sistemas complexos que integram as instalações físicas da indústria com a computação e englobam máquinas inteligentes capazes de trocar informações de forma independente, trazendo melhorias fundamentais para os processos industriais envolvidos na fabricação, engenharia, uso de material, cadeia de suprimentos e gestão do ciclo de vida (Vidal \& Másculo, 2011). Neste conceito, os processos 
físicos são modelados no ambiente virtual, onde é possível interagir e criar uma network entre pessoas, máquinas, insumos de produção e sistemas de computação em toda a cadeia de valor, formando uma rede capaz de autogerir-se. Para isso, os sistemas são compostos pela combinação de tecnologias de uma maneira nova, embora ainda seja um conceito em desenvolvimento (Vidal e Másculo, 2011).

Segundo o BCG (The Boston Consulting Group, 2015), a Indústria 4.0 é um modelo composto por nove avanços tecnológicos fundamentais:
a. Big Data e Analytics;
b. Robôs autônomos;
c. Simulação;
d. Sistemas de Integração Vertical e Horizontal;
e. Internet das Coisas;
f. Cyber-segurança;
g. Nuvem;
h. Fabricação aditiva;
i. Realidade aumentada.

O termo Indústria 4.0 possui outras nomenclaturas, tais como "Fábricas Inteligentes", "Indústria Inteligente" ou "Produção Avançada". O modelo da Indústria Inteligente traz um grande avanço no modelo de negócio das organizações, o que reflete em ganhos expressivos em toda a cadeia de valor dos produtos e serviços, de forma a otimizar a logística e torná-la cada vez mais eficiente, exigindo uma integração on-line de fornecedores, máquinas e clientes (Freitas et al.,2016).

É importante destacar que, ao contrário das outras revoluções industriais observadas e diagnosticadas a posteriori, essa é a primeira vez que os acontecimentos estão sendo previstos como tendências. Assim, muitos fatores condicionantes ainda não são observados e nem toda a valoração de impactos é estimada (Hermann, Pentek e Otto, 2015). Um dos maiores impactos futuros da indústria 4.0 é a criação de novos modelos de negócios. Apesar disso, poucas empresas estão capacitadas para enfrentar todos os desafios oriundos desta mudança de uma só vez. Por outro lado, há milhares de empresas que devem se envolver no processo de multiplicação dessas novas tecnologias progressivamente, de acordo com suas trajetórias, capacitações e técnicas (CNI, 2016).

Empresas que desejam percorrer a trajetória para a Indústria 4.0 devem avaliar suas capacidades e adaptar suas estratégias de forma a implementá-la nos cenários apropriados. Ultrapassar estes desafios envolve o cumprimento de alguns requisitos como: responder às questões de segurança e proteção digital, padronização das interfaces de comunicação, processos e organização do trabalho, disponibilidade de força de trabalho capacitada, inserção das pequenas e médias empresas, formação e desenvolvimento profissional, base tecnológica, investigação e investimentos (European Parliament, 2016).

Todavia, um dos desafios mais críticos enfrentados pela Indústria 4.0 está relacionado com as pessoas. Os novos cenários trazem implicações significativas na natureza do trabalho, já que transformam a concepção, a fabricação, a operação dos produtos e serviços nos sistemas de produção (The Boston Consulting Group, 2015). Assim torna-se necessária a preparação e envolvimento do aspecto humano dentro de todo o processo de desenvolvimento e implantação destas ferramentas.

Como a Indústria 4.0 se trata de uma teoria emergente, a investigação desta pesquisa tem um caráter exploratório e visa contribuir para o aprimoramento da teoria sobre sua aplicação no Brasil. A investigação aborda o risco que uma população que vem envelhecendo pode representar, em termos de capacitação, para otimização da cadeia de valor da indústria brasileira através da implementação das tecnologias do modelo de 
Indústria 4.0. Isto posto, o presente artigo tem como objetivo compreender e avaliar os impactos que esta dinâmica pode causar na implementação do modelo de Indústria 4.0 no Brasil, utilizando o PMBOK e matriz de risco, que comtempla identificação, análise e resposta aos riscos do projeto.

\section{REFERENCIAL TEÓRICO}

O conhecimento da indústria brasileira sobre tecnologias digitais e sua incorporação à produção, precondições para o avanço da Indústria 4.0, ainda é pouco difundido (CNI, 2016a). Uma empresa deve ser entendida como a interligação de três sistemas - o social, o estrutural e o tecnológico. Tais premissas são norteadas ao indicar os principais elementos aderentes à Indústria 4.0 e analisar a influência do fator humano durante a implantação de novas tecnologias. Esta integração traz diversos impactos aos processos de negócios e, por muitas vezes, negligenciar o fator humano acaba criando barreiras e atrapalhando benefícios destas novas tecnologias (Maximiano, 2002).

Para Maximiano (2002), há a interligação de três sistemas dentro de uma empresa:

- Sistema social: os funcionários da organização comportam-se como pessoas, organismos, e não como máquinas. O comportamento e interesses individuais, de grupos e dos integrantes da organização interagem e são, muitas vezes, conflitantes. Neste ambiente, o relacionamento informal influência o comportamento organizacional;

- Sistema estrutural: tem a estrutura hierárquica organizacional, normas e regulamentos como importantes instrumentos para ação disciplinadora dos grupos, porém eles podem se tornar barreiras na disseminação da informação e do conhecimento em organizações de gerenciamento complexos;

- Sistema tecnológico: acrescenta, além dos maquinários utilizados para o desempenho das atividades esperadas pelos gestores, a tecnologia moderna.

Contudo, os principais impactos da Indústria 4.0 ocorrem na força de trabalho e na necessidade de as pessoas aperfeiçoarem suas competências para lidar com todas as novas tecnologias e garantir sua empregabilidade, o que faz dessa maior exigência de qualificação juntamente com as mudanças demográficas já em curso - como o envelhecimento da população - os maiores desafios sociais a serem superados (Schwab, 2016; Hecklau, 2016). É por isso que, assim como sugerido por Buhr (2015), é "imprescindível que olhemos com mais atenção para esse aspecto, para que seja possível identificar onde estão os riscos, mas também as oportunidades para o progresso e a inovação social".

Nesse sentido, a Gestão de Riscos, é recomendada para que as organizações a incorporem aos seus respectivos planejamentos, subsidiando a tomada de decisão em todos os departamentos e atividades, incluindo estratégias, operações, processos, funções, projetos, produtos, serviços e ativos (Ferreira, 2013; FNQ, 2014; IBCG,2007). A Gestão de Riscos contempla os processos envolvidos na identificação, análise e resposta aos riscos do projeto (PMBOK, 2017).

\section{METODOLOGIA}

O estudo de caso é um trabalho de caráter empírico que investiga um dado 
fenômeno dentro de um contexto real contemporâneo por meio de análise aprofundada de um ou mais objetos de análise (Cauchick, 2010). Neste artigo, considera as operações, as tecnologias e o fator humano aderentes à Indústria 4.0 sob a perspectiva dos sistemas social, estrutural e tecnológico, com um olhar sociotécnico que traça uma correlação de interdependência entre as necessidades tecnológicas e humanas nas organizações.

Os dados são obtidos através de duas publicações de 2016 referentes à pesquisa sobre digitalização e utilização de tecnologias digitais realizada pela empresa Price Waterhouse Coopers (PwC), onde são analisadas mais de 2.000 empresas participantes dos nove maiores setores industriais de 26 países, incluindo o Brasil (PwC, 2016), e do relatório publicado pela Confederação Nacional da Indústria (CNI) intitulado "Desafios para Indústria 4.0 no Brasil", onde é apresentado o panorama da digitalização da indústria brasileira (CNI, 2016a).

A preparação e as coletas de dados para este estudo de caso são realizadas por meio de entrevistas, realizadas pelas próprias organizações supracitadas, seguindo seus respectivos protocolos para a condução das pesquisas. Com base nestes, é realizada uma triangulação dos resultados apresentados, a fim de identificar as convergências dos estudos quanto à identificação dos fatores que representam ameaças à implementação da Indústria 4.0 no Brasil dentro da abordagem sociotécnica.

Para construir o modelo a ser analisado, também são utilizados dados referentes ao comportamento da dinâmica populacional brasileira, extraídos de relatórios do Instituto Brasileiro de Geografia e Estatística (IBGE) e do site Population Pyramid, que monitora a evolução da pirâmide populacional do Brasil e do mundo desde 1950 e simula as previsões para os próximos 80 anos.

No que tange as análises, são identificados alguns riscos que a realidade brasileira representa para implementação da Indústria 4.0 no país. A análise é feita utilizando as diretrizes do PMBOK (2017), descritas na Tabela 1, através da utilização de um exemplo de matriz de risco construído e aplicado pelo Ministério da Transparência e Controladoria Geral da União (CGU) indicado na Tabela 2.

Tabela 1: Fases do processo de gerenciamento de riscos de um projeto.

\begin{tabular}{|c|c|c|}
\hline 1 & Planejar o gerenciamento dos riscos & $\begin{array}{l}\text { Definição de como conduzir as atividades de gerenciamento de riscos do } \\
\text { projeto. }\end{array}$ \\
\hline 2 & Identificar os ricsos & $\begin{array}{l}\text { Identificação dos riscos individuais do projeto, bem como fontes de risco geral } \\
\text { do projeto, e documentar suas características. }\end{array}$ \\
\hline 3 & Realizar a análise qualitativa dos riscos & $\begin{array}{l}\text { Priorização de riscos individuais do projeto para análise ou ação posterior, } \\
\text { através da avaliação da sua probabilidade de ocorrência e impacto, assim } \\
\text { como outras características. }\end{array}$ \\
\hline 4 & Realizar a análise quantitativa dos riscos & $\begin{array}{l}\text { Analisar numericamente o efeito combinado dos riscos individuais } \\
\text { identificados no projeto e outras fontes de incerteza nos objetivos gerais do } \\
\text { projeto. }\end{array}$ \\
\hline 5 & Planejar respostas aos riscos & $\begin{array}{l}\text { Desenvolver alternativas, selecionar estratégias e acordar ações para lidar com } \\
\text { a exposição geral de riscos, e também tratar os riscos individuais do projeto. }\end{array}$ \\
\hline 6 & Implementar respostas aos riscos & Implementar planos acordados de resposta aos riscos. \\
\hline 7 & Monitorar os riscos & $\begin{array}{l}\text { Monitorar a implementação de planos acordados de resposta aos riscos, } \\
\text { acompanhar riscos identificados, identificar e analisar novos riscos, e avaliar a } \\
\text { eficácia do processo de risco ao longo do projeto. }\end{array}$ \\
\hline
\end{tabular}

Fonte: PMBOK (2017). 
Tabela 2 - Matriz de Riscos (Probabilidade e Impacto)

\begin{tabular}{|c|c|c|c|c|c|c|}
\hline \multirow{5}{*}{ 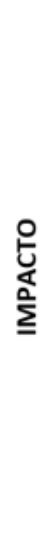 } & 올온운 & $\begin{array}{c}10 \\
\mathrm{RM}\end{array}$ & $\begin{array}{c}20 \\
\mathrm{RM}\end{array}$ & $\begin{array}{l}50 \\
\text { RA }\end{array}$ & $\begin{array}{l}80 \\
R E\end{array}$ & $\begin{array}{l}100 \\
\mathrm{RE}\end{array}$ \\
\hline & $\frac{\stackrel{9}{4}}{4} \infty$ & $\begin{array}{c}8 \\
R B\end{array}$ & $\begin{array}{c}16 \\
\mathrm{RM}\end{array}$ & $\begin{array}{l}40 \\
\text { RA }\end{array}$ & $\begin{array}{l}64 \\
\text { RA }\end{array}$ & $\begin{array}{l}80 \\
R E\end{array}$ \\
\hline & 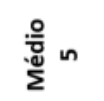 & $\begin{array}{c}5 \\
\mathrm{RB}\end{array}$ & $\begin{array}{l}10 \\
\mathrm{RM}\end{array}$ & $\begin{array}{l}25 \\
\mathrm{RM}\end{array}$ & $\begin{array}{l}40 \\
\text { RA }\end{array}$ & $\begin{array}{l}50 \\
\text { RA }\end{array}$ \\
\hline & $\frac{0}{\sqrt[x]{\pi}} N$ & $\begin{array}{c}2 \\
\mathrm{RB}\end{array}$ & $\begin{array}{c}4 \\
R B\end{array}$ & $\begin{array}{c}10 \\
\mathrm{RM}\end{array}$ & $\begin{array}{l}16 \\
\mathrm{RM}\end{array}$ & $\begin{array}{c}20 \\
\mathrm{RM}\end{array}$ \\
\hline & 莺 这 & $\begin{array}{c}1 \\
\mathrm{RB}\end{array}$ & $\begin{array}{c}2 \\
R B\end{array}$ & $\begin{array}{c}5 \\
\mathrm{RB}\end{array}$ & $\begin{array}{c}8 \\
R B\end{array}$ & $\begin{array}{c}10 \\
\mathrm{RM}\end{array}$ \\
\hline & & $\begin{array}{c}\text { Muito Baixa } \\
1\end{array}$ & $\begin{array}{c}\text { Baixa } \\
2\end{array}$ & $\begin{array}{c}\text { Média } \\
5\end{array}$ & $\begin{array}{c}\text { Alta } \\
8\end{array}$ & $\begin{array}{c}\text { Muito Alta } \\
10\end{array}$ \\
\hline & & \multicolumn{5}{|c|}{ PROBABILIDADE } \\
\hline
\end{tabular}

Fonte: CGU (2018).

Por fim, são confrontados os impactos do envelhecimento populacional na População Economicamente Ativa (PEA) e outros impactos nos âmbitos tecnológico e estrutural, com as probabilidades de ocorrência de cenário atribuídas experimentalmente pelos autores, para melhor compreender como estes fatores podem afetar a concretização do modelo na indústria brasileira. $\mathrm{O}$ objetivo do gerenciamento de riscos nesse sentido é maximizar a probabilidade e o impacto dos efeitos positivos e minimizar dos efeitos negativos.

\section{ANÁLISE DOS DADOS COLETADOS}

Os estudos utilizados como base para esta metodologia apontam a capacitação como um dos grandes desafios para implementação da Indústria 4.0 no Brasil, conforme descrito nos itens 4.1 e 4.2 .

\subsection{RESUltados PUbLiCAdOS PELA CNI}

Conforme o relatório publicado pela CNI, a incorporação da digitalização à atividade industrial fez o conceito de Indústria 4.0 ser também referenciado como a $4^{\mathrm{a}}$ Revolução Industrial. O desenvolvimento da Indústria 4.0 no Brasil envolve desafios como os investimentos em equipamentos que incorporem as novas tecnologias, adaptação de layouts, processos e formas de relacionamento entre as empresas ao longo da cadeia produtiva, criação de novas especialidades e desenvolvimentos de competências, entre outras (CNI, 2016b).

A Tabela 3 identifica 10 tecnologias digitais e revela o conhecimento da indústria brasileira sobre essas tecnologias. A pesquisa realizada pela CNI em 2.225 empresas brasileiras, sendo 910 pequenas, 815 médias e 500 grandes, de 29 setores da indústria de transformação e extrativa, aponta que $42 \%$ das empresas desconhecem a importância das tecnologias digitais e 52\% não utilizam nenhuma das tecnologias digitais que compunham a lista de opções da pesquisa (CNI, 2016b). 
Tabela 3 - Resultado da pesquisa realizada pela CNI

\begin{tabular}{lcc} 
Automação digital sem sensores & 11 & 3 \\
\hline Automação digital com sensores para controle de processo & 27 & 20 \\
\hline Monitoramento e controle remoto da produção com sistemas do tipo MES e SCADA & 7 & 14 \\
\hline $\begin{array}{l}\text { Automação digital com sensores com identificação de produtos e condições } \\
\text { operacionais, linhas flexíveis }\end{array}$ & 8 & 21 \\
\hline $\begin{array}{l}\text { Sistemas integrados de engenharia para desenvolvimento de produtos e } \\
\text { manufatura de produtos }\end{array}$ & 19 & 25 \\
\hline Manufatura aditiva, prototipagem rápida ou impressão 3D & 5 & 9 \\
\hline $\begin{array}{l}\text { Simulações/análise de modelos virtuais (Elementos Finitos, Fluidodinâmica } \\
\text { Computacional, etc.) para projeto e comissionamento }\end{array}$ & 5 & 15 \\
\hline Coleta, processamento e análise de grandes quantidades de dados (big data) & 9 & 11 \\
\hline Utilização de serviços em nuvem associados ao produto & 6 & 12 \\
\hline $\begin{array}{l}\text { Incorporação de serviços digitais nos produtos (“Internet das Coisas” ou } \\
\text { Product Service Systems) }\end{array}$ & 4 & 9 \\
\hline Projetos de manufatura por computador CAD/CAM (2) (3) & 30 & 15 \\
\hline Nenhuma das listadas & 31 & 39 \\
\hline Não sabe/ não respondeu & & 3 \\
\hline
\end{tabular}

Fonte: CNI (2016b).

Outro dado importante é que $31 \%$ das empresas entrevistadas não responderam ou não souberam opinar se utilizavam alguma das tecnologias listadas, revelando alto nível de desconhecimento sobre o tema, conforme é mostrado na tabela de resultados abaixo (CNI, 2016b). Assim, a CNI entende que as novas formas de produção decorrentes da Indústria 4.0 exigem profissionais com formação distinta das existentes, exigindo equipes multidisciplinares, com elevado nível de conhecimento técnico e com capacidade de interação de diferentes áreas de conhecimento (CNI, 2016b).

\subsection{Resultados Publicados Pela PwC}

$\mathrm{Na}$ concepção da $\mathrm{PwC}$, a Indústria 4.0 compreende a digitalização e integração dos processos verticalmente em toda a organização, desde o desenvolvimento e a compra de produtos, até a fabricação, logística e serviços. A pesquisa realizada pela empresa em 2016, considerada por ela como o maior levantamento dessa natureza já realizado no mundo, aponta que, das empresas brasileiras entrevistadas, apenas $9 \%$ se consideram avançadas em níveis de digitalização. Porém, esse percentual pode chegar a $72 \%$ em 2020 , conforme as expectativas das entrevistadas, o que representa um aumento significativo na utilização de tecnologias digitais no país (PwC, 2016).

Dentre as principais conclusões da pesquisa, destaca-se o foco em pessoas, cultura e segurança para impulsionar a transformação, onde os entrevistados, tanto as empresas globais quanto as brasileiras, consideram que seu maior desafio não é a implementação da tecnologia certa, e sim a falta de cultura digital e de habilidades em sua organização. Para os entrevistados, o investimento em tecnologias adequadas é importante, mas o sucesso ou 
fracasso vai depender de diversos fatores relacionados a pessoas, pois as empresas precisam desenvolver uma cultura digital robusta e certificar-se de que a mudança é impulsionada por uma liderança clara da alta administração, além da necessidade de atrair, reter e treinar a força de trabalho para atuar em um ambiente digital dinâmico (PwC, 2016).

\subsection{Panorama da PopulaÇÃo economicamente ativa (PEA) No Brasil}

A análise mais recente de contingente ocupado divulgada pelo IBGE, publicada em fevereiro de 2019, traz dados da Pesquisa Nacional por Amostra de Domicílios Contínua (PNAD Contínua) e informa que, no $4^{\circ}$ trimestre de 2018, 12,6\% do contingente ocupado são jovens de 18 a 24 anos, adultos nas faixas de 25 a 39 anos e 40 a 59 anos de idade representa $77,8 \%$ e que os idosos correspondem a $8,1 \%$ (Figura 1). Por pessoas ocupadas entende-se que, no período de referência, pessoas exercendo ao menos uma hora completa em trabalho remunerado em dinheiro, produtos, mercadorias ou benefícios (moradia, alimentação, roupas, treinamento etc.), ou em trabalho sem remuneração direta em ajuda à atividade econômica de membro do domicílio ou parente que reside em outro domicílio, ou, ainda, as que possuem trabalho remunerado do qual estavam temporariamente afastadas nessa semana de referência da pesquisa (IBGE, 2019).

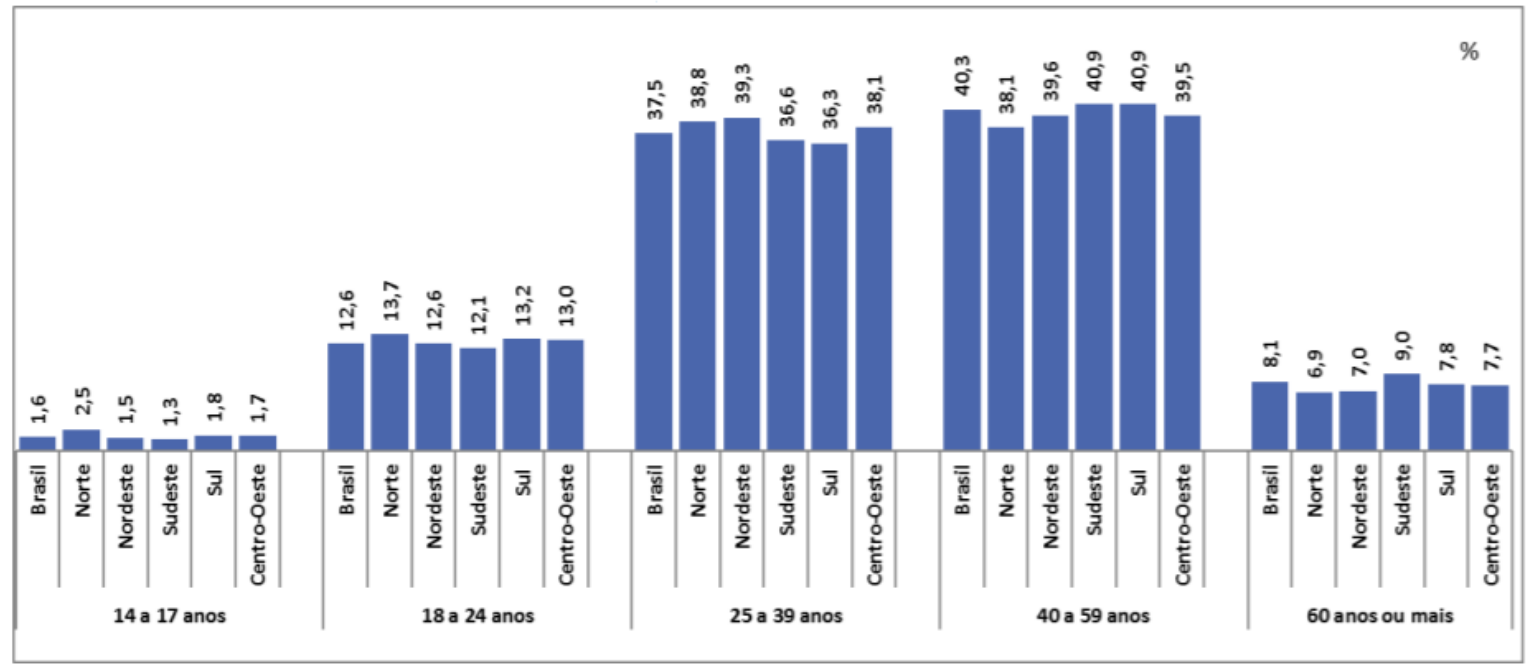

Figura 1 - Distribuição percentual de pessoas de 14 anos ou mais de idade, ocupadas na semana de referência, por grupos de idade, segundo as Grandes Regiões - $4^{\circ}$ trimestre/2018

Fonte: IBGE (2019).

Os gráficos da Figura 2 mostram uma evolução da dinâmica populacional desde o ano 2000 até a projeção para o ano 2040 através de pirâmides etárias. A camada da população com menor idade, parte inferior do gráfico, tende a ficar cada vez mais reduzida, consequência de fatores como o planejamento familiar para controle de natalidade e redução das taxas de mortalidade. Além disso, devido aos avanços na medicina dos últimos anos, a população está vivendo por mais anos, enquanto a mortalidade infantil teve forte redução (Araújo e Alves, 2000). Segundo o IBGE, a expectativa de vida ao nascer vem aumentando, atingindo 80 anos para mulheres e 73 anos para homens em 2019. 


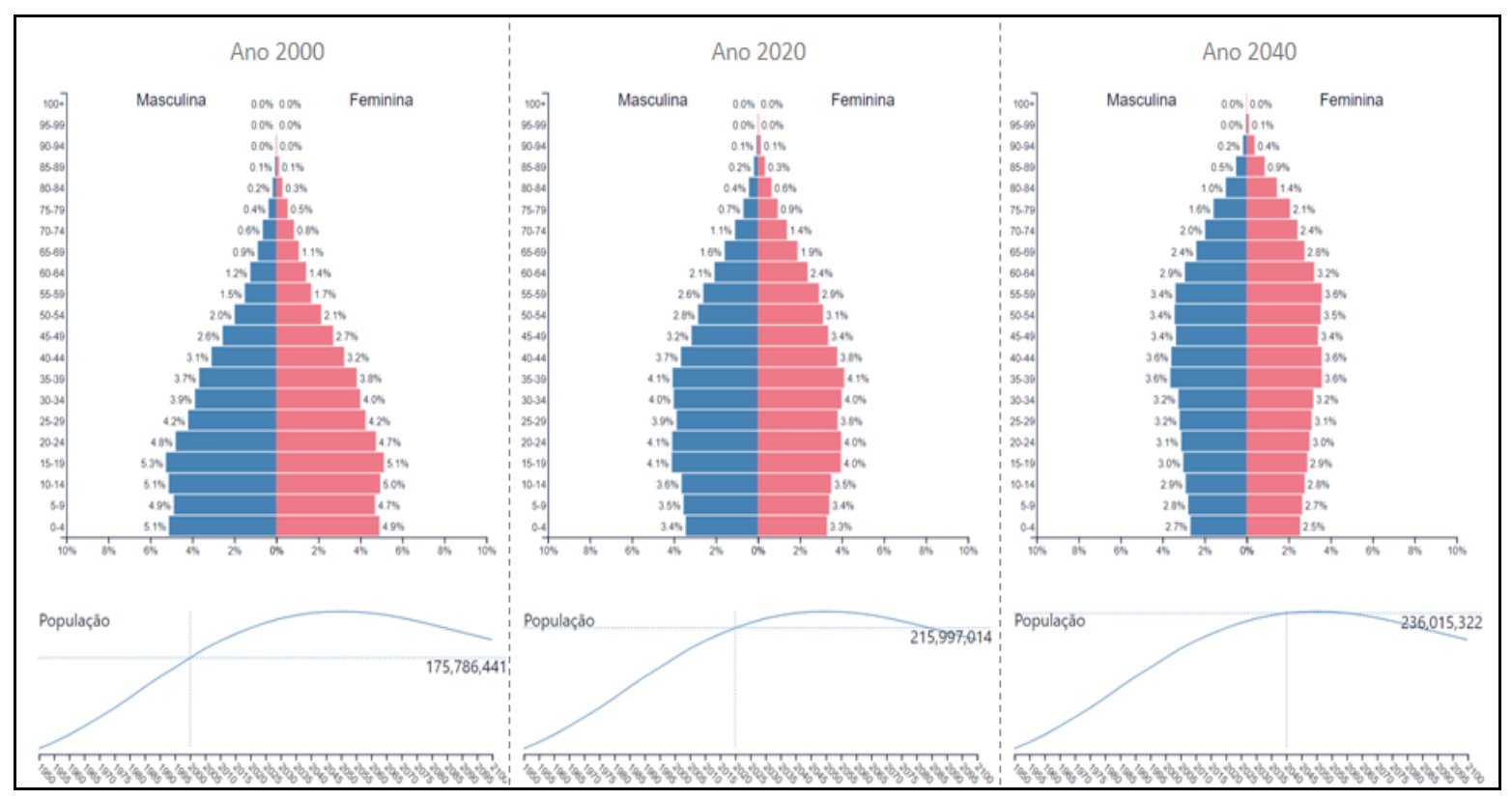

Figura 2 - Dinâmica populacional dos anos de 2000, 2020 e 2040.

Fonte: Population Pyramid (2019).

Observa-se assim que o Brasil passa por um processo de transição demográfica. Em Oliveira (2016), há uma discussão sobre como as tendências das taxas brutas de natalidade e mortalidade podem acarretar, no início dos anos 2040, a redução no volume populacional do Brasil tais transformações na dinâmica demográfica podem impactar não só o volume, mas também a estrutura etária e, consequentemente, a população em idade ativa, com a redução no número de crianças e o aumento no segmento idoso, como sugerem os gráficos na Figura 2 .

\section{ANÁLISE DE RISCO}

Os dados publicados pela PNAD (IBGE, 2019) ao final de 2018, apontam que a maior concentração da população ocupada está entre indivíduos de 18 a 59 anos de idade, representando $90,4 \%$ da população ocupada no país. Idosos ocupados representam apenas $8,1 \%$. Conforme a pirâmide populacional apresentada na Figura 2, os indivíduos entre 16 e 59 anos de idade representam atualmente $65,6 \%$ da população total nacional, e a projeção para o ano de 2040 é que estes passem a representar 59,8\% da população. Esta redução de aproximadamente 6\% deve representar um impacto grande na PEA, visto que menor quantidade de pessoas deve continuar tendo que arcar com $90 \%$ do trabalho no país. Consequentemente espera-se que os idosos ocupados não sejam mais apenas $8,1 \%$, esta parcela da PEA deve crescer.

É possível identificar riscos associados a diferentes aspectos da implementação da Indústria 4.0 nas organizações. Sob o ponto de vista social, verifica-se exigência dos trabalhadores por um alto nível de conhecimento técnico e capacidade de interação de diferentes áreas de conhecimento. Observando-se a idade mais avançada e sabendo que a curva de aprendizado é desigual para indivíduos de diferentes faixas etárias, um risco associado pode ser a dificuldade de aprendizado e, consequentemente, prejuízo e atraso na adaptação da produção aos novos tempos (Miranda, 2016). De acordo com a matriz de riscos da Tabela 2, assumindo probabilidade de ocorrência entre 5 e 8 e impacto acima de 5, atribui-se um risco para essa dimensão.

Observando aspectos associados ao sistema estrutural, sabe-se que na Indústria 4.0 
os fluxos de informações são essenciais para o funcionamento da produção. Assim, a limitada infraestrutura de telecomunicação como acesso à banda larga e rede móvel, por exemplo, podem ser entraves para seu funcionamento. Aqui, entende-se que uma vez que os avanços nesta área são implementados, todos os processos passam a depender deles. Caso a comunicação pare de funcionar, a cadeia produtiva pode parar completamente, afetando todo o negócio. Considerando que as tecnologias aplicadas à área de telecomunicações no Brasil ainda são bastante deficientes, supõe-se que este risco tem probabilidade alta (8) e impacto muito alto (10), resultando em um risco é extremo.

No campo tecnológico, a Indústria 4.0 abre oportunidades para o desenvolvimento de soluções empresariais onde parte do desafio é a identificação de oportunidades e o entendimento do ambiente em que as dificuldades são menores, consequentemente as chances de desenvolvimento são maiores. Neste caso, um risco reside na necessidade de customizar soluções para diferentes clientes. Esta prática exige conhecimentos avançados em tecnologias específicas como software e integração de sistemas, por exemplo. A possibilidade de que a capacidade técnica da empresa não acompanhe as novas tecnologias, sugere assumir probabilidade média (5) com impacto muito alto (10), já que novamente a produção fica seriamente comprometida. Assim, considera-se este risco alto.

Após essa atribuição dos graus de risco, tem-se que faixa de classificação do risco deve ser considerada na definição da atitude da organização em relação ao tratamento. Segundo a PGR/CGU, apetite a risco é o "nível de risco que a empresa está disposta a aceitar". É importante que o apetite a risco do processo organizacional seja estabelecido no início do processo de gerenciamento de riscos. Uma vez definido, a unidade declara que: todos os riscos cujos níveis estejam dentro da(s) faixa(s) de apetite a risco podem ser aceitos, e uma possível priorização para tratamento deve ser justificada; todos os riscos cujos níveis estejam fora da(s) faixa(s) de apetite a risco serão tratados e monitorados, e uma possível falta de tratamento deve ser justificada. A Tabela 4 mostra um exemplo de quais ações adotar em cada classificação de risco.

\begin{tabular}{l|l}
$\begin{array}{c}\text { Tabela 4 - Atitude perante o risco para cada classificação. } \\
\text { Classificação }\end{array}$ & \multicolumn{1}{c}{ Ação necessária } \\
\hline Risco Baixo & $\begin{array}{l}\text { Nível de risco dentro do apetite a risco, mas é possível que } \\
\text { existam oportunidades de maior retorno que podem ser } \\
\text { exploradas assumindo-se mais riscos, avaliando a relação custo } \\
\text { x benefício, como diminuir o nível de controles. }\end{array}$ \\
\hline Risco Médio & $\begin{array}{l}\text { Nível de risco dentro do apetite a risco. Geralmente nenhuma } \\
\text { medida especial é necessária, porém requer atividades } \\
\text { de monitoramento espećficas e atenção da unidade na } \\
\text { manutenção de respostas e controles para manter o risco } \\
\text { nesse nível, ou reduzi-lo sem custos adicionais. }\end{array}$ \\
\hline Risco Alto & $\begin{array}{l}\text { Nível de risco além do apetite a risco. Qualquer risco nesse } \\
\text { nível deve ser comunicado ao dirigente máximo da unidade e } \\
\text { ter uma ação tomada em período determinado. Postergação } \\
\text { de medidas só com autorização do dirigente máximo da } \\
\text { unidade. }\end{array}$ \\
\hline \multirow{2}{*}{ Risco Extremo } & $\begin{array}{l}\text { Nível de risco muito além do apetite a risco. Qualquer risco } \\
\text { nesse nível deve ser objeto de Avaliação Estratégica (seção } \\
\text { 4.I I), comunicado ao Comitê de Gestão Estratégica e ao } \\
\text { dirigente máximo da unidade e ter uma resposta imediata. } \\
\text { Postergação de medidas só com autorização do Comitê de } \\
\text { Gestão Estratégica. }\end{array}$
\end{tabular}

Fonte: CGU (2018). 
Esta análise indica que um projeto para implementação da Indústria 4.0 no mercado brasileiro demanda um aprofundamento da abordagem sociotécnica do modelo, considerando, tanto a forte tendência de mudança na estrutura da PEA, quanto à necessidade de desenvolvimento das novas competências profissionais que o modelo exige.

\section{CONCLUSÃO}

As inovações tecnológicas podem afetar processos produtivos e, frequentemente, costumam substituir o trabalho, em particular o menos qualificado. Assim como no exterior, no Brasil, a tecnologia e a inovação têm modificado constantemente velhos padrões de organização da produção, criando novas formas de produzir os mesmos produtos ou produtos antes inexistentes (Ford, 2016).

Observam-se nos últimos anos importantes transformações na estrutura produtiva brasileira, com significativas consequências sobre o emprego. A Indústria 4.0 trouxe maior exposição aos requisitos de competitividade necessários para ganhar novos mercados, o que resulta na perda de importância de algumas atividades menos competitivas e no aumento da participação de outras na estrutura produtiva. Todo esse processo gera modificações, é claro, na forma como se distribui e se organiza o emprego no Brasil (Fiuza-Moura et. Al, 2015).

A evolução da dinâmica populacional provoca o menor ingresso de indivíduos jovens em populações que passam a viver períodos mais longos. Este fenômeno tem impacto direto na estrutura da população ocupada e, portanto, no perfil dos trabalhadores a serem submetidos às mudanças provocadas pelos desdobramentos da Indústria 4.0. Observa-se ainda que a Gestão de Risco tem importante papel na identificação e avaliação destas mudanças e, consequentemente, na criação de alternativas e execução das ações necessárias.

$\mathrm{O}$ ambiente empresarial exige a utilização de ferramentas que tragam vantagem competitiva no mercado. As ferramentas de tecnologia de informação são essenciais para que as empresas consigam sobreviver neste novo ambiente. A obtenção de conhecimento da organização pode ser facilitada oferecendo aos seus colaboradores oportunidades como cursos técnicos para atender a necessidades específicas, reformulação de cursos já existentes nas áreas de engenharia, cursos de gestão da produção multidisciplinar com ênfase em Indústria 4.0 e incentivo de programas de competências tecnológicas nas empresas (CNI, 2016). O sucesso das medidas depende do envolvimento dos diversos setores da empresa e da definição clara de quais os objetivos alcançar.

O intercâmbio tecnológico e comercial com outros países também pode ser fundamental para o acesso ao conhecimento. Assim, políticas de apoio à Indústria 4.0 devem fomentar esses tipos de intercâmbios para absorver, da forma mais eficiente possível, as tecnologias estrangeiras e viabilizar uma maior capacidade competitiva (Schwab, 2016).

Como continuidade, para futuras pesquisas, sugere-se aprofundar a investigação sobre os riscos identificados e a condução de pesquisas empíricas, por meio de entrevistas com gestores brasileiros, visando a compreender se estes possuem visões semelhantes ou distintas às apresentadas neste estudo. Pode-se, ainda, realizar pesquisas em que os impactos no trabalho sejam contrapostos a outros impactos, como políticos e econômicos, de forma a obter um debate mais rico e amplo sobre a temática.

O estudo realizado também deixa claro que os investimentos em novas tecnologias se tornam obsoletos quando implementados sem haver mudanças estruturais conjuntamente. Faz-se necessário capacitar pessoal, modernizar processos e tornar-se adaptável a mudanças de paradigmas, pois não o objetivo é implantar modernidades de forma que as organizações possam absorvê-las e aceitá-las completamente. 


\section{REFERÊNCIAS BIBLIOGRÁFICAS}

[1] ARAÚJO, T. C. N.; ALVES, M. I. C. Perfil da população idosa no Brasil. Textos sobre Envelhecimento, Rio de Janeiro, RJ, UNATI, v. 3. n. 3, 2000.

[2] BLANCHET, M.; RINN, T.; THADEN, G.V.; THIEULLOY, G.D. Industry4.0: The new industrial revolution How Europe will succeed. Munich: Think Act. Roland Berger, 2014.

[3] BUHR, D. Social innovation policy for Industry 4.0. Friedrich-Ebert-Stiftung, Division for Social and Economic Policies, 2015.

[4] CAUCHICK, M. O método do estudo de caso na Engenharia de Produção. Rio de Janeiro: Elsevier, 2010.

[5] CNI, CONFEDERAÇÃO NACIONAL DA INDÚSTRIA. Desafios para a indústria 4.0 no Brasil. Brasília: CNI, 2016a.

[6] CNI, CONFEDERAÇÃO NACIONAL DA INDÚSTRIA. Sondagem Especial $\mathbf{n}^{\mathbf{0}} \mathbf{6 6}$. Indústria 4.0. Brasília: CNI, 2016b.

[7] EUROPEAN PARLIAMENT. Industry 4.0. 2016.

[8] FERREIRA, R. de O.; LIMA, A.J.Q.; LIMA, J.B.A.; TREINTA, F.T. Contribuição do "Círculo de Stakeholders na Etapa de Comunicação e Consulta da ISO 31000 em uma Empresa do Setor de Energia. XXXIII Encontro Nacional de Engenharia de Produção (ENEGEP), 2013.

[9] FORD, M. Robôs: A Ameaça de Um Futuro sem Emprego. 2016.

[10] FREITAS, M.M.B.C.; FRAGA, M.A.F.; SOUZA, G.P.L. Logística 4.0: Conceitos e aplicabilidade - uma pesquisa ação em uma empresa de tecnologia para o mercado automobilístico. Caderno PAIC, v.17, n.1, p.237-261, 2016.

[11] FUNDAÇÃO NACIONAL DA QUALIDADE (FNQ). Gestão de Risco. 2014

[12] HECKLAU, F.; GALEITZKE, M.; FLACHS, S.; KOHL, H. Holistic approach for human resource management in Industry 4.0. Procedia CIRP, 2016.

[13] HERMANN, M.; PENTEK, T.; OTTO B. Design Principles for Industrie 4.0 Scenarios: A Literature Review. Working Paper $N^{\circ}$. 01, 2015.

[14] IBGC. Guia de Orientação para Gerenciamento de Riscos Corporativos. São Paulo: IBGC, 2007.

[15] IBGE. Pesquisa Nacional por Amostra de Domicílios Contínua Terceiro Trimestre de 2018. Rio de Janeiro: IBGE, 2019.

[16] KAGERMANN, H.; WAHLSTER, W.; HELBIG, J. Recommendations for implementing the strategic initiative industrie 4.0: final report of the industrie 4.0. Berlin : Forschungsunion, 2013.

[17] MINISTÉRIO DA TRANSPARÊNCIA E CONTROLADORIA-GERAL DA UNIÃO (CGU). Metodologia de Gestão de Riscos. Brasília: CGU, 2018.

[18] MIRANDA, G.M.D.; MENDES, A. DA C.G.; SILVA, A.L.A. DA. Population aging in Brazil: current and future social challenges and consequences. Revista Brasileira de Geriatria e Gerontologia, v. 19, n. 3, p.507-519. 2016. 
[19] OLIVEIRA, A. T. R. Envelhecimento populacional e políticas públicas: desafios para o Brasil no século XXI. Revista Brasileira de Geografia Econômica, v.8, p.1-20, 2016.

[20] POPUlatiOn PYRAMID. Pirâmides Populacionais do Mundo desde 1950 até 2100. Disponível em: <http://www.populationpyramid.net/pt/brasil>. Acessado em 23 de maio de 2019.

[21] PRICE WATERHOUSE COOPERS (PWC). Indústria 4.0: Digitalização como vantagem competitiva no Brasil. Brasil: PWC, 2016

[22] SCHWAB, K. The Fourth Industrial Revolution. Genebra: World Economic Forum, 2016.

[23] THE BOSTON CONSULTING GROUP. Industry 4.0 - The Future of Productivity and Growth in Manufacturing Industries. Boston: The BCG, 2015.

[24] VIDAL M.C; MÁSCULO, F.S. Ergonomia: trabalho adequado e eficiente. Rio de Janeiro: Editora Campus, 2011. 